Prof. T.T. Cloete

Departement Afrikaans-Nederlands en ATLW

\title{
NUWE DIGBUNDELS XIV
}

(Bespreck in Tydskrif.vir Geesteswelenskappe 23, 4:304 e.v. Desember 1983)

BARNARD, Erika. Animato, Perskor-Uitgewery, Johannesburg, 1982. 39 bl. R9,95.

BELCHER, R.K. Halleluja Paternoster. Perskor-Uitgewery, Johannesburg, 1982. 57 bl. R9,95.

BELCHER, R.K. Kinge in 'n geelhoutboom. Perskor-Uitgewery, Johanncslsurg. 1982. 42 bl. R9,95.

CARSTENS, Annalisé. Skanse. John Malherbe, Kaapstad, 1982. 56 bl. R4,00.

CLOE'ГE, 'Г.'Г.,7uksiaposisie. Talelberg-Uitgewers, Kaapstad, 1982. $117 \mathrm{bl}$. R10,50.

DE LANGE, Johann. Akwarelle van die dors. Human en Rousseau, Kaapstad, Pretoria, Johannesburg, 1982. 57 bl., prys nie vermeld nie.

DE VOS, Annesu. Om ury uit te stap. Talelberg-Uitgewers, Kaapstad, 1982. 67 , prys nie vermeld nie.

EKSTEEN, Louis. Skuilhoek. Perskor-Uitgewery, Johannesburg, 1982. 88 bl. $R 9,95$.

ENGELBRECHT, Herman. Kruispunt. Uitgewery Oranje, Pretoria, 1981. 59 bl. R6,80.

ENGELBRECHT, Theunis. 'n Gedig is so onskuldig soos 'n eier. PerskorUitgewery, Johannesburg, 1982. 59 bl. R9,95.

EYBERS, E. Bestand. Human en Rousseau, Kaapstad, Pretoria, Johannesburg, 1982, 51 bl. R7,50.

HUGO, Daniël. Korte metle. Human en Rousseau, Kaapstad, Pretoria, Johannesburg, 1981. 41 bl. R6,50.

KIRSCH, Olga. Afskeide. Human en Rousseau, Kaapstad, Pretoria, Johannesburg, 1982. 37 bl. R5,95.

IJEACH, C.P. Borgtog vir 'n nuwe Adam. Perskor-Uitgewery, Johannesburg. 1982. 68 bl. R9,95.

LETOIT, André. Suburbia. Perskor-Uitgewery, Johannesburg, 1982. $59 \mathrm{bl}$. R9,95.

PHILANDER, P.J. Venster. Perskor-Uitgewery, Johannesburg, 1982. 71 bl. R9,95

PRETORIUS, S.J. Serusiet. Perskor-Uitgewery, Johannesburg, 1982 (foutief aangedui as 1983). $103 \mathrm{bl}$. R9,95.

RALl, Henk. Proefskrif. Perskor-Uitgewery, Johannesburg, 1982. $69 \mathrm{bl.}$ R9.95. 
SCHMID'T, Casper. Pelgrims in aspiek. Perskor-Uitgewery, Johannesburg, 1982. 49 bl., prys nie vermeld nie.

SNYDERS, Peter. 'n Ordinary mens. Tafelberg-Uitgewers, Kaapstad, 1982. 46 bl., prys nie vermeld nie.

SPIES, Lina. Oorstaanson. Human en Rousseau, Kaapstad, Pretoria, Johannesburg, 1982. 88 bl. R10,50.

VAN DER MER WE, Daan. Saad uit die septer. P.J. de Villiers, Bloemfontein. I98I. 60 bl. R4,95.

VAN HEERDEN, Ernst. Kanse op 'n wrak. Tafelberg-Uitgewers, Kaapstad, 1982. $78 \mathrm{bl}$, prys nie vermeld nie.

VILJOEN, Hein. Waterkrislal. Perskor-Uitgewery, Johannesburg, 1982. 48 bl. R9,95.

WESSELS, Everwyn. Miknes. Perskor-Uitgewery, Johannesburg, 1982. 73 bl. R9.95.

WILLEMSE, Rudolph. Kweekskool. Perskor-Uitgewery, Johannesburg, 1982. $37 \mathrm{bl}$., prys nie vermeld nie. 